OPEN ACCESS

Edited by:

Robin Polt,

The University of Arizona,

United States

Reviewed by:

Shekher Mohan,

Manchester University, United States

Jian Cheng,

Soochow University, China

*Correspondence:

Bing-Qiao Zhao

bingqiaoz@fudan.edu.cn

Wenying Fan

wenyingf@fudan.edu.cn

Specialty section:

This article was submitted to

Neuropharmacology,

a section of the journal

Frontiers in Neuroscience

Received: 18 July 2018

Accepted: 22 March 2019

Published: 05 April 2019

Citation:

Luo H, Wei L, Lu L, Kang L,

Cao $Y$, Yang $X$, Bai $X$, Fan $W$ and

Zhao B-Q (2019) Transfusion

of Resting Platelets Reduces Brain

Hemorrhage After Intracerebral

Hemorrhage and tPA-Induced

Hemorrhage After Cerebral Ischemia.

Front. Neurosci. 13:338.

doi: 10.3389/fnins.2019.00338

\section{Transfusion of Resting Platelets Reduces Brain Hemorrhage After Intracerebral Hemorrhage and tPA-Induced Hemorrhage After Cerebral Ischemia}

\author{
Haiyu Luo, Lixiang Wei, Lu Lu, Lijing Kang, Yongliang Cao, Xing Yang, Xiaofei Bai, \\ Wenying Fan* and Bing-Qiao Zhao*
}

Department of Translational Neuroscience, Jing'an District Centre Hospital of Shanghai, State Key Laboratory of Medical Neurobiology and MOE Frontiers Center for Brain Science, Institutes of Brain Science, Fudan University, Shanghai, China

Background: Exacerbated blood-brain barrier (BBB) damage is related with tissue plasminogen activator (tPA)-induced brain hemorrhage after stroke. Platelets have long been recognized as the cellular orchestrators of primary haemostasis. Recent studies have demonstrated further that platelets are required for supporting intact mature blood vessels and play a crucial role in maintaining vascular integrity during inflammation. Therefore, we sought to investigate whether platelets could reduce tPA-induced deterioration of cerebrovascular integrity and lead to less hemorrhagic transformation.

Methods: Mice were subjected to models of collagenase-induced intracerebral hemorrhage $(\mathrm{ICH})$ and transient middle cerebral artery (MCA) occlusion. After $2 \mathrm{~h}$ of MCA occlusion, tPA (10 mg/kg) was administered as an intravenous bolus injection of $1 \mathrm{mg} / \mathrm{kg}$ followed by a $9 \mathrm{mg} / \mathrm{kg}$ infusion for $30 \mathrm{~min}$. Immediately after tPA treatment, mice were transfused with platelets. Hemorrhagic volume, infarct size, neurological deficit, tight junction and basal membrane damages, endothelial cell apoptosis, and extravascular accumulation of circulating dextran and lgG, and Evans blue were quantified at $24 \mathrm{~h}$.

Results: Platelet transfusion resulted in a significant decrease in hematoma volume after $\mathrm{ICH}$. In mice after ischemia, tPA administration increased brain hemorrhage transformation and this was reversed by resting but not activated platelets. Consistent with this, we observed that tPA-induced brain hemorrhage was dramatically exacerbated in thrombocytopenic mice. Transfusion of resting platelets ameliorated tPA-induced loss of cerebrovascular integrity and reduced extravascular accumulation of circulating serum proteins and Evans blue, associated with improved neurological functions after ischemia. No changes were found for infarct volume. Inhibition of platelet 
receptor glycoprotein VI (GPVI) blunted the ability of platelets to attenuate tPA-induced BBB disruption and hemorrhage after ischemia.

Conclusion: Our findings demonstrate the importance of platelets in safeguarding BBB integrity and suggest that transfusion of resting platelets may be useful to improve the safety of tPA thrombolysis in ischemic stroke.

Keywords: cerebral hemorrhage, tPA, blood-brain barrier integrity, platelets, GPVI

\section{INTRODUCTION}

Acute cerebral ischemia is a common form of stroke with high rates of mortality and disability (Roth et al., 2015). Intravenous (IV) injection of the tissue plasminogen activator (tPA) is the only medical therapy approved by the United States Food and Drug Administration (FDA) to treat acute ischemic stroke (Jauch et al., 2013), due to its thrombolytic activity and its ability of restoring circulation to the brain (Hacke et al., 1995). However, thrombolysis induces a markedly increased risk of the intracerebral hemorrhage (ICH), which is fatal in ischemic stroke patient after tPA treatment (Whiteley et al., 2012). However, the appropriate treatment with thrombolysis avoiding hemorrhage in acute ischemic stroke patients has neither been determined. Accumulating clinical and experimental evidence have revealed that tPA exacerbated ischemic endothelial injury and blood-brain barrier (BBB) disruption (Suzuki et al., 2016), which is well recognized the precursor event to hemorrhagic transformation. Thus, improvement of the $\mathrm{BBB}$ integrity might be a beneficial strategy for the treatment of thrombolysis-associated ICH.

Platelets are anucleate myeloid blood cells that have a multitude of physiological functions (Ghoshal and Bhattacharyya, 2014). They circulate in blood vessels without forming interactions with non-activated vascular endothelium under normal physiologic conditions (Rasche, 2001). The roles of platelets play in adhering and aggregating at the sites of injured vascular structures, first reported by Bizzozero, have long been recognized as the primary hemostatic function of platelets (Ribatti and Crivellato, 2007). With the expanding breadth of knowledge about platelets, increasing evidence on platelet biology have uncovered a large variety of new functions for platelets. Over the past years, studies show that platelet transfusion prevents hemorrhage in intratumor blood vessels and preserves the vascular integrity independently of forming hemostatic plugs at sites of vascular injury (Ho-Tin-Noe et al., 2008, 2011). More recently, platelets are recognized as central actors of many other pathophysiological processes such as protecting the vasculature against leakage and safeguarding developing vessels, lymphatics, as well as the vascular integrity under inflammatory conditions (Goerge et al., 2008; Lee and Bergmeier, 2016). Meanwhile, it has also been discovered that in thrombocytopenic mice, more hemorrhagic foci are seen around the infarction area after middle cerebral artery occlusion compared with normal mice (Goerge et al., 2008). Platelet has been continuously focused on both experimental stroke and clinical stroke, and there's increasing evidence suggesting us much more function of platelets should be highlighted besides of the clear aspect on a thromboembolic basis (Mancuso and Santagostino, 2017).

Many academic institutions have developed their own urgent care pathways for managing tPA-associated hemorrhage including the use of platelets (Goldstein et al., 2010). However, there's few reliable research data available to provide evidence for clinical treatment with platelets for thrombolysis-associated ICH. In this study, we investigated the role of platelets on tPA-induced $\mathrm{BBB}$ disruption and hemorrhage after cerebral ischemia. We found that platelet transfusion significantly blocked tPA-associated loss of cerebrovascular integrity, and protected BBB permeability, consequently reduced tPA-induced cerebral hemorrhage.

\section{MATERIALS AND METHODS}

\section{Animal Stroke Model}

All experimental procedures were approved by the Animal Care and Use Committee of Institutes of Brain Science, Fudan University. All animals used were adult male C57BL/6J mice (Shanghai SLAC Laboratory Animal Co., Shanghai, China) weighing 21 to $28 \mathrm{~g}$ at 8 to 10 weeks of age. The ICH model was induced by a striatum injection of $0.025 \mathrm{U}$ collagenase type VII (Sigma-Aldrich, St Louis, MO, United States) in $0.5 \mu \mathrm{L}$ saline with a 26-gauge needle inserted into the striatum through a burr hole on the skull (Matsushita et al., 2011). After removing the needle, $6 \times 10^{8}$ washed platelets were transfused IV into mice (Gros et al., 2015). Transient focal cerebral ischemia was induced as previously reported (Wang et al., 2013). In brief, mice were subjected to transient occlusion of the right middle cerebral artery (MCAO) with a 7.0 siliconized filament through the external carotid artery. The filament was removed to allow reperfusion after occlusion for $45 \mathrm{~min}$. Anesthesia was induced with 1 to $1.5 \%$ isoflurane in a mixture of $30 \%$ oxygen and $70 \%$ nitrogen. During the operations, the body temperature was maintained at $37 \pm 0.5^{\circ} \mathrm{C}$ using a heating pad. Successful MCA occlusions and reperfusion were confirmed by Laser Doppler flowmetry (Perimed, Stockholm, Sweden), and only animals with a $>70 \%$ reduction in cerebral blood flow after MCAO and a $>50 \%$ of baseline cerebral blood flow after filament withdrawal were included in this study. At $2 \mathrm{~h}$ after MCAO, tPA $(10 \mathrm{mg} / \mathrm{kg}$, Actilyse, Mannheim, Germany) or PBS was administered as an intravenous bolus injection of $1 \mathrm{mg} / \mathrm{kg}$ followed by a $9 \mathrm{mg} / \mathrm{kg}$ infusion for $30 \mathrm{~min}$ with a syringe infusion pump (World Precision Instruments, Sarasota, FL, United States). D-glucose $(6 \mathrm{~mL} / \mathrm{kg}$ at $50 \% \mathrm{wt} / \mathrm{vol}$, Sigma-Aldrich) was used to increase 
the extent of cerebral hemorrhage by an intraperitoneal injection 15 min before MCAO as described previously (Wang et al., 2013). Mice were transfused with $6 \times 10^{8}$ washed platelets immediately after tPA treatment. Mice that died after MCAO surgery were excluded from the end-point analyses. The mortality rates in each study were as follows: among the collagenase-induced $\mathrm{ICH}$ mice ( $n=7$ per group), the mortality rate was $12.5 \%(1 / 8)$ in $\mathrm{ICH}$ group, $0 \%(0 / 7)$ in $\mathrm{ICH}+$ platelets or tyrode buffer group; among the ischemic mice, the mortality rate was $10.9 \%(5 / 46)$ in vehicle group, $22.6 \%(12 / 53)$ in tPA group, $14.9 \%(10 / 67)$ in tPA + platelets group, $24.1 \%(13 / 54)$ in tPA + tyrode buffer group, $20 \%(2 / 10)$ in tPA + activated platelets group, 20\% (4/20) in tPA + IgG group, $42.8 \%$ (12/28) in tPA + anti-GPIb $\alpha$ group, $23.8 \%$ $(5 / 21)$ in $\mathrm{tPA}+\mathrm{JAQ} 1$-platelets group.

\section{Platelet Preparation}

Washed platelets were prepared from WT mice as described (Cazenave et al., 2004). Donor mice were anesthetized by isoflurane, and whole blood was collected from the retroorbital venous plexus and sampled in acid-citrate-dextrose (ACD: 2.5\% trisodium citrate, $2 \% \mathrm{D}$-glucose and $1.5 \%$ citric acid) [at 1 (ACD): 9 (blood) ratio]. To obtain washed platelets, the sampled blood was first centrifuged at $800 \mathrm{rpm}, 4 \mathrm{~min}$, then the plateletrich fraction and part of the red blood cells were collected with supplement of $1 \mu \mathrm{L}$ prostacyclin $\left(\mathrm{PGI}_{2}, 1 \mathrm{mg} / \mathrm{mL}\right.$, SigmaAldrich) and further centrifuged at $1200 \mathrm{rpm}, 4 \mathrm{~min}$. The plateletrich plasma (PRP) was collected and added with $1 \mu \mathrm{L} \mathrm{PGI}_{2}$. After centrifugation (2600 rpm, $4 \mathrm{~min}$ ), the isolated platelets were washed twice with HEPES-Tyrode's buffer and resuspended to a concentration of $6 \times 10^{9}$ platelets $/ \mathrm{mL}$.

\section{Platelet Depletion and Platelet Activation}

Platelet depletion was induced by an intravenous injection of $1 \mu \mathrm{g} / \mathrm{g}$ bodyweight of the platelet-depleting antibody (GPIb $\alpha$ antibody; Emfret Analytics, Würzburg, Germany) or matched non-immune isotype antibody (Emfret Analytics) to mice immediately after tPA treatment. Platelet counts were evaluated by flow cytometry. Platelets in the presence of $2 \mathrm{mmol} / \mathrm{L}$ ethylenediaminetetraacetic acid (EDTA) to avoid aggregation were stimulated with human thrombin (1 units $/ \mathrm{mL}$, SigmaAldrich), an activator of platelets for $10 \mathrm{~min}$ at $37^{\circ} \mathrm{C}$. Hirudin (2 units/mL, Sigma-Aldrich), a specific inhibitor of thrombin was added to stop the reaction (Ho-Tin-Noe et al., 2008). Eight mice were evaluated per group for measurements of hemorrhagic volume, BBB permeability and flow cytometry.

\section{Flow Cytometry}

Platelet-rich plasma was prepared by centrifugation of anticoagulated blood as previously described (Vaiyapuri et al., 2012). For flow cytometry, $10 \mu \mathrm{L}$ of this suspension sample was incubated with $1 \mu \mathrm{L}$ of fluorescein isothiocyanate (FITC) conjugated CD61 MoAb (BD Pharmingen, San Diego, CA). Immediately afterward, $39 \mu \mathrm{L}$ of PBS was added with gentle mixing. After $30 \mathrm{~min}$ of incubation at room temperature in the dark, $450 \mu \mathrm{L}$ of PBS was added and the samples were analyzed on a FACSCalibur flow cytometer (BD Bioscience) using CellQuest (BD Bioscience) and FlowJo (TreeStar) software (Arroyo et al., 2001).

\section{Inhibitor Studies}

To inhibit GPVI function, washed platelets were incubated with the inhibitory antibody JAQ1 (100 $\mu \mathrm{g} / \mathrm{mL}$, Emfret Analytics) in vitro, after $15 \mathrm{~min}$, the unbound JAQ1 antibody was washed away (Boulaftali et al., 2013). The JAQ1-pretreated platelets were transfused into mice ( $n=8$ per group) as described above.

\section{Quantification of Cerebral Hemorrhage}

Intracerebral hemorrhage was quantified with a spectrophotometric assay as previously described (Wang et al., 2013). Mice ( $n=8$ per group) were anesthetized and perfused transcardially with ice-cold PBS at $24 \mathrm{~h}$ after MCAO. The ischemic hemisphere of each mouse was homogenized and centrifuged at $13,000 \mathrm{rpm}$ for $30 \mathrm{~min}$ with Drabkin reagent (500 $\mu \mathrm{L}$, Sigma-Aldrich). Optical density of supernatants was measured at $540 \mathrm{~nm}$ by spectrophotometry (Thermo Fisher Scientific BioMate 3S; Thermo Fisher Scientific, Waltham, MA, United States). A reference curve was generated by blending different known volumes of fresh mouse blood into normal brain samples. Hemorrhage volume was expressed in equivalent units by comparison with this reference curve.

\section{BBB Permeability Evaluation}

At $23 \mathrm{~h}$ after MCAO ( $n=8$ per group), Evans blue dye ( $4 \%$ in PBS, $4 \mathrm{~mL} / \mathrm{kg}$, Sigma-Aldrich) injections were given intravenously and allowed to circulate for $1 \mathrm{~h}$. The ischemic hemispheres were weighted and placed in formamide for $72 \mathrm{~h}$ in the dark. The amount of extravasated Evans blue dye was evaluated by spectrophotometry (Thermo Fisher Scientific BioMate 3S; Thermo Fisher Scientific) at $620 \mathrm{~nm}$ (Cai et al., 2015).

\section{Detection of Extravascular Dextran and $\lg G$}

A tracer FITC-labeled dextran was used to estimate the in vivo BBB permeability as previously described (Xu et al., 2017). Briefly, vascular leakage was analyzed after IV injection of $40 \mathrm{kDa}$ FITC-dextran (50 $\mu \mathrm{L}$ of $100 \mathrm{mg} / \mathrm{mL}$, Sigma-Aldrich) circulating for $2 \mathrm{~min}$. The brains were quickly removed and postfixed in $4 \%$ paraformaldehyde at $4^{\circ} \mathrm{C}$ overnight, and then immersed $48 \mathrm{~h}$ in $30 \%$ sucrose in PBS. $50 \mu \mathrm{m}$-thick coronal brain sections were immunostained with goat anti-CD31 (AF3628, R\&D Systems, Minneapolis, MN, United States) and incubated with Alexa Fluor 594-conjugated donkey anti-goat IgG (Invitrogen). The images of extravascular dextran fluorescence were processed by Image J software and the dextran leakage was quantified as a percentage of dextran ${ }^{+}$area. Extravascular IgG was measured by incubating cerebral tissue sections with a combination of antibodies of goat anti-CD31 (AF3628, R\&D Systems), Alexa Fluor 488-conjugated donkey anti-mouse IgG and Alexa Fluor 594 donkey anti-goat IgG (Invitrogen) (Xu et al., 2017). The images were digitized using a $40 \times$ objective in the peri-infarct area and the immunofluorescent intensity of extravascular IgG 
was quantified using the ImageJ Integrated Density analysis measurement tool. Five mice were used in each group.

\section{Measurement of Infract Volume}

Mice ( $n=5$ per group) were sacrificed by overdose of chloral hydrate and the brains were cut into $1-\mathrm{mm}$ thick coronal slices using a rodent brain matrix. Eight selected sections were stained for $30 \mathrm{~min}$ in a $2 \%$ solution of triphenyl-2, 3, 4-tetrazoliumchloride (TTC, Sigma-Aldrich). Sections were digitalized and the volume of ischemic brain injury was measured blindly using the ImageJ software (Fan et al., 2016).

\section{Western Blotting}

The ischemic hemispheric brain tissues and matching tissues from sham-operated mice ( $n=5$ per group) were removed and used for western blot as previously described (Wang et al., 2013). The primary antibodies were: rabbit anti-collagen IV (ab6586, Abcam, Cambridge, MA, United States), rabbit antiZO-1 (617300, Invitrogen, Camarillo, CA, United States), rabbit anti-occludin (ab167161, Abcam), and rabbit anti- $\beta$-actin (4970, Cell Signaling Technology, Beverly, MA, United States).

\section{Immunohistochemistry}

After transcardial perfusion/fixation with ice-cold $4 \%$ paraformaldehyde and immersed in $30 \%$ sucrose in PBS at $4^{\circ} \mathrm{C}$ overnight, the brain tissues ( $n=5$ per group) were cut into $20 \mu \mathrm{m}$ sections. Immunohistochemistry was performed as previously described (Xu et al., 2017). The primary antibodies used were: goat anti-CD31 (AF3628, R\&D Systems, Minneapolis, MN, United States), rabbit anti-collagen IV (ab19808, Abcam), rabbit anti-ZO-1 (617300, Invitrogen, Camarillo, CA, United States), rabbit anti-occludin (ab404700, Abcam), rabbit anti-fibrinogen (AP00766PU-N, Acris Antibodies, CA, United States). The secondary antibodies used were: Alexa Fluor 594-conjugated donkey anti-goat IgG, Alexa Fluor 488-conjugated donkey anti-rabbit IgG (all from Invitrogen). Images were obtained using an Olympus BX 51 microscope and an Olympus FV1000 confocal microscope. For quantification of the positive cells, 6 randomly selected non-overlapping images $(40 \times$ objective) in the peri-infarct area were digitized and analyzed using ImageJ software.

\section{Immunofluorescence Double Staining for CD31 and TUNEL}

TUNEL staining was performed on brain cryosections according to the manufacturer's manual of in situ cell death detection kit (Roche Diagnostics, Mannheim, Germany) followed by CD31 immunofluorescent staining as described (Bruns et al., 2000). We determined the percentage of TUNEL-positive endothelial cells in the peri-infarct area using six different microscopic fields per section at $400 \times$ magnification and five mice were evaluated per group.

\section{Behavioral Measurements}

At $24 \mathrm{~h}$ after MCAO, mice ( $n=10$ per group) were examined and scored blindly using the modified Neurological Severity Scores
(mNSS) (Chen et al., 2001). The mNSS is a composite of motor, sensory, beam balance and reflex tests by a grading scale of 0 (normal score) to 18 (maximal deficit score).

\section{Statistical Analysis}

Data are expressed as the mean \pm SD and were assessed with one-way analysis of variance after the Bonferroni multiple comparison test. When two groups were compared, values were analyzed using unpaired 2-tailed Student $t$-test. Behavior data were analyzed using Kruskal-Wallis test followed by the Dunn multiple comparison test. Differences were considered statistically significant with $p$-values $<0.05$.

\section{RESULTS}

\section{Transfusion of Resting Platelets Reduces Brain Hemorrhage After ICH and Cerebral Ischemia}

Using the collagenase-induced ICH model, we found that transfusion of resting platelets significantly reduced cerebral hematoma volume compared with vehicle treatment at $24 \mathrm{~h}$ after ICH (Figure 1A). To investigate the role of platelets on tPAmediated hemorrhage after ischemic stroke, we subjected mice to $45 \mathrm{~min}$ of focal cerebral ischemia using the MCAO model. As reported, infusion of tPA $2 \mathrm{~h}$ after MCAO resulted in a significant increase in hemorrhage (Wang et al., 2013). Treatment with resting platelets successfully blocked tPA-induced hemorrhage, whereas thrombin-activated (degranulated) platelets failed to reduce the hemorrhage (Figures $\mathbf{1 B}, \mathbf{C}$ ). These data suggest that mediators released from platelet granules might be critical for preventing tPA-induced hemorrhage. A recent study also shows that platelet secretion is associated with cerebral hemorrhage after ischemia (Deppermann et al., 2017). Moreover, in our study, combination treatment with resting platelets and tPA did not affect infarct volume (Figure 1E) and intravascular fibrin deposits (Supplementary Figure S1B) compared with the tPA alone group. Previous study showed that platelet depletion by anti-GPIb $\alpha$ antibody alone increased the hemorrhage compared with the mice with normal platelet count after MCAO (Zhao et al., 2009). To further confirm the role of platelets on tPAinduced hemorrhage, we induced thrombocytopenia in mice by platelet depletion using an anti-GPIb $\alpha$ antibody (Supplementary Figure S1A). These experiments showed that treatment with tPA led to more severe hemorrhage in thrombocytopenic mice compared with mice with normal platelet counts (Figures 1B,C). In addition, the tPA-treated mice transfused with platelets had better neurological scores than those without platelet transfusion (Figure 1D).

\section{Resting Platelets Block tPA-Induced Increase in BBB Permeability and Preserve Vascular Integrity After Cerebral Ischemia}

To evaluate the BBB damage, we quantified the perivascular IgG deposits, leakage of dextran $(\mathrm{MW}=40,000 \mathrm{Da})$, and Evans blue 


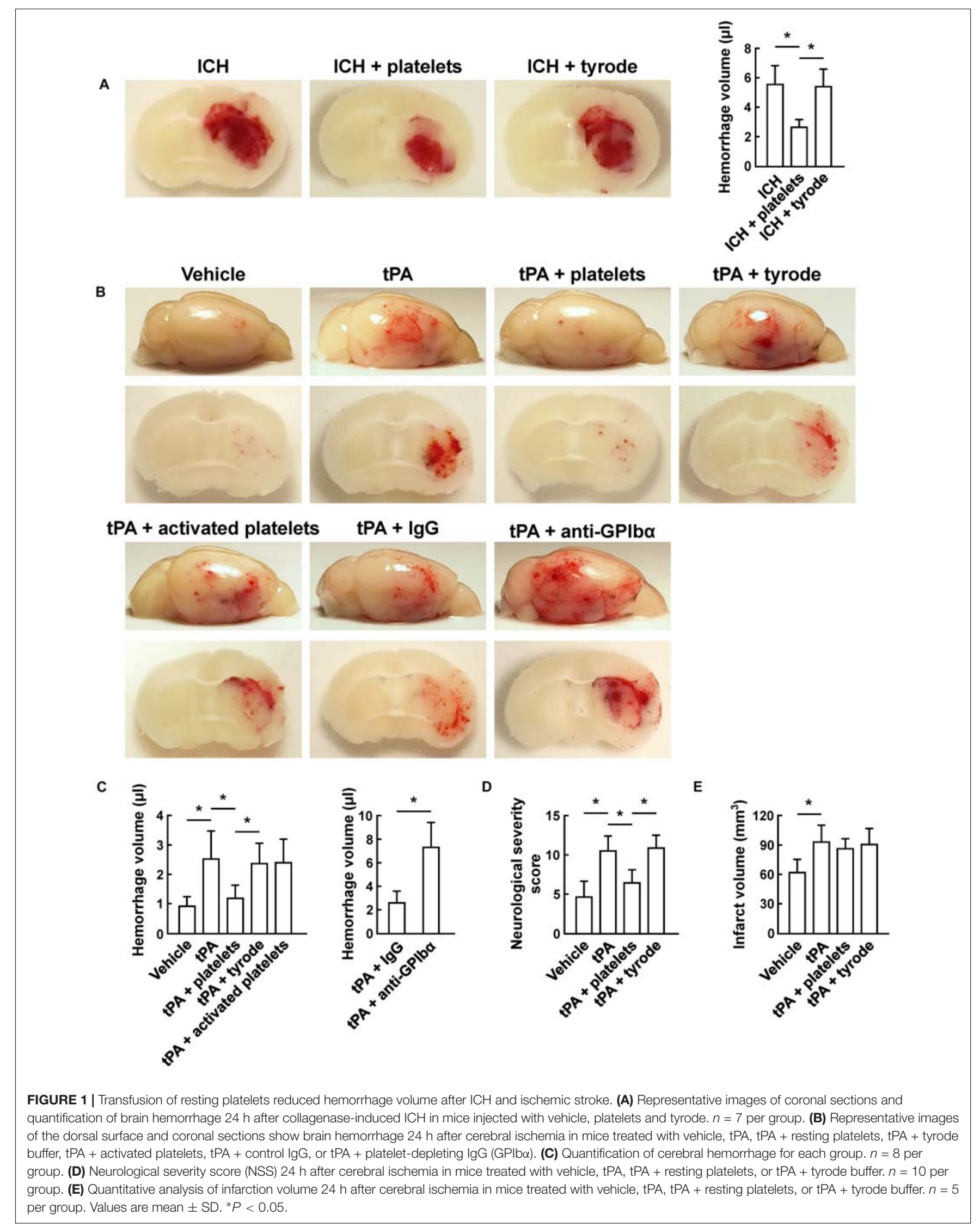



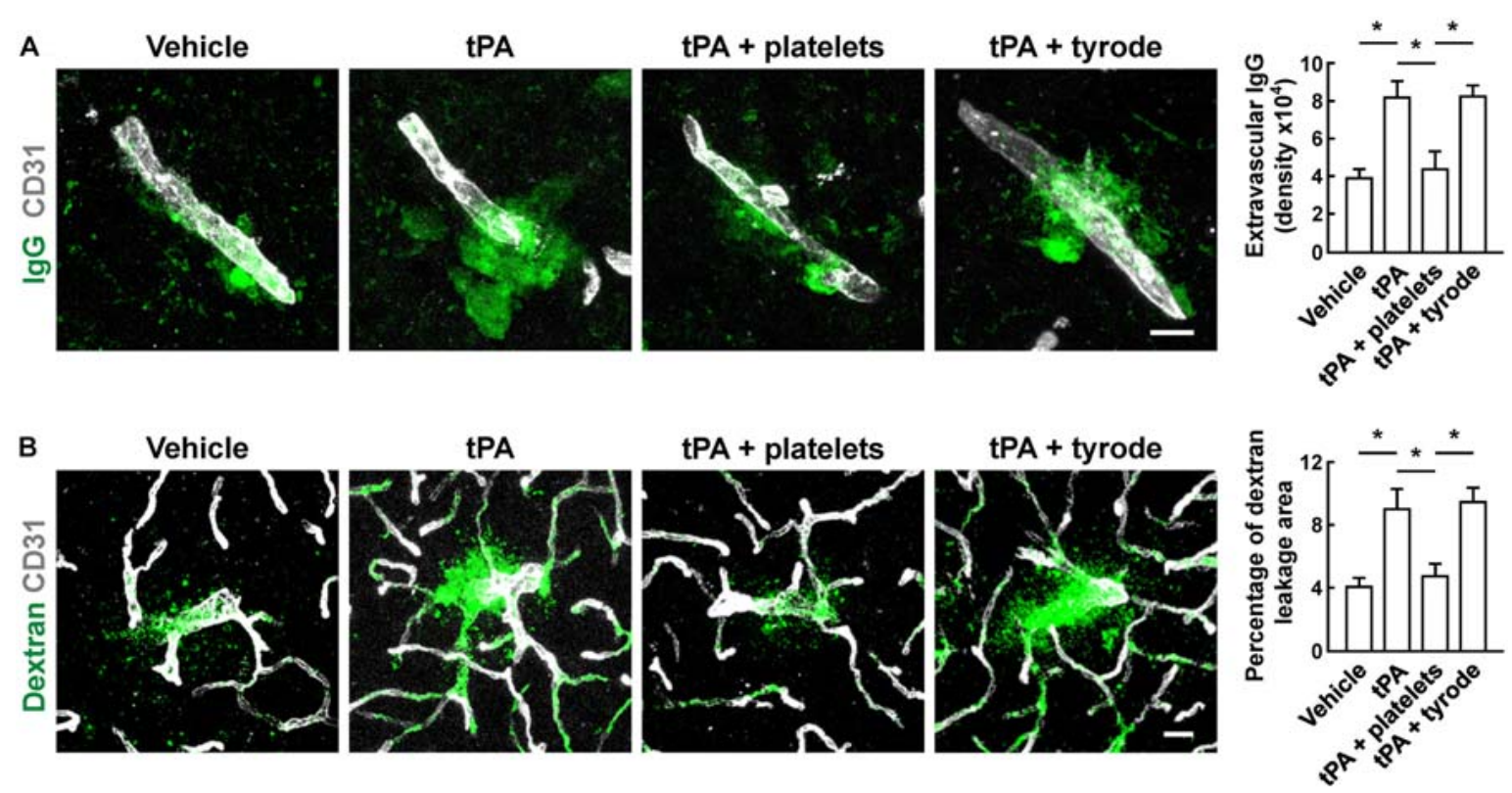

C

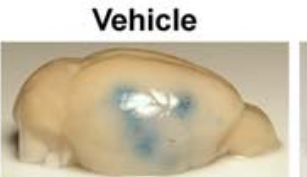

tPA
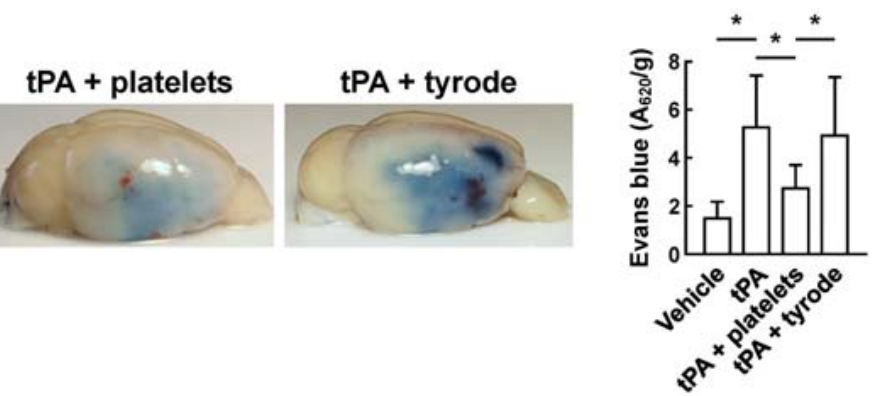

FIGURE 2 | Transfusion of resting platelets reduced tPA-induced BBB disruption after cerebral ischemia. (A) Representative images and quantitative analysis of IgG leakage $24 \mathrm{~h}$ after cerebral ischemia in mice treated with vehicle, tPA, tPA + resting platelets, or tPA + tyrode buffer. Bar $=10 \mu \mathrm{m}$. $n=5$ per group. (B) Representative images and quantitative analysis of extravascular dextran fluorescence in mice treated with vehicle, tPA, tPA + resting platelets, or tPA + tyrode buffer. Bar $=20 \mu \mathrm{m}$. At $24 \mathrm{~h}$ after stroke, mice were given an intravascular injection of 40,000 Da FITC-labeled dextran, and brain sections were stained with CD31. $n=5$ per group. (C) Representative photographs and quantification of Evans blue dye extravasation $24 \mathrm{~h}$ after cerebral ischemia. $n=8$ per group. Values are mean \pm SD. $* P<0.05$.

infiltration to the brain parenchyma. The results showed that tPA exacerbated the disruption of the BBB, but these effects were reversed by platelet transfusion (Figures $\mathbf{2 A}-\mathbf{C}$ ), which revealed that resting platelets resulted in a protective role of BBB function. Furthermore, we investigated the role of resting platelets on tPA-mediated degradation of $\mathrm{BBB}$ components after stroke. The dual staining for the $\mathrm{BBB}$ tight junction proteins including ZO-1 and occludin, each combined with $\mathrm{CD} 31$, indicated significant increases in $\mathrm{ZO}-1^{+}$coverage and occludin $^{+}$coverage in platelets-treated mice (Figures $3 \mathbf{A}, \mathbf{B}$ ). Mice with platelet transfusion also exhibited increase in the major vascular basal membrane protein collagen IV, as shown by immunostaining of brain microvessels (Figure 3C). Together with these findings, western blot analysis showed that treatment of mice with platelets remarkably preserved the loss of these three proteins (Figure 3D). We next determined the effects of platelets on vascular injury based on the data above using double staining of CD31 (endothelial cells) and TUNEL (apoptotic cells). tPA critically enhanced endothelial cell apoptosis after cerebral ischemia, whereas resting platelets substantially diminished tPAmediated vascular damage (Figure 4).

\section{Resting Platelets Prevent tPA-Induced Hemorrhage and Reduce BBB Damage via GLYCOPROTEIN VI (GPVI)-Dependent Manner}

Glycoprotein VI is the major collagen receptor mediating primary adhesion of platelets to the vascular wall (Nieswandt and Watson, 2003). Recent evidence exposed that the vascular protective role of platelets was independent of their ability to form thrombi (Goerge et al., 2008; Ho-Tin-Noe et al., 2011), and inhibition of GPVI partially abolished the protective function of platelets on vascular integrity during inflammation (Boulaftali et al., 2013; Gros et al., 2015). We speculated that whether GPVI act primarily in the maintenance of cerebrovascular integrity by preventing hemorrhage and regulating $\mathrm{BBB}$ permeability after cerebral ischemia. We first incubated resting platelets 


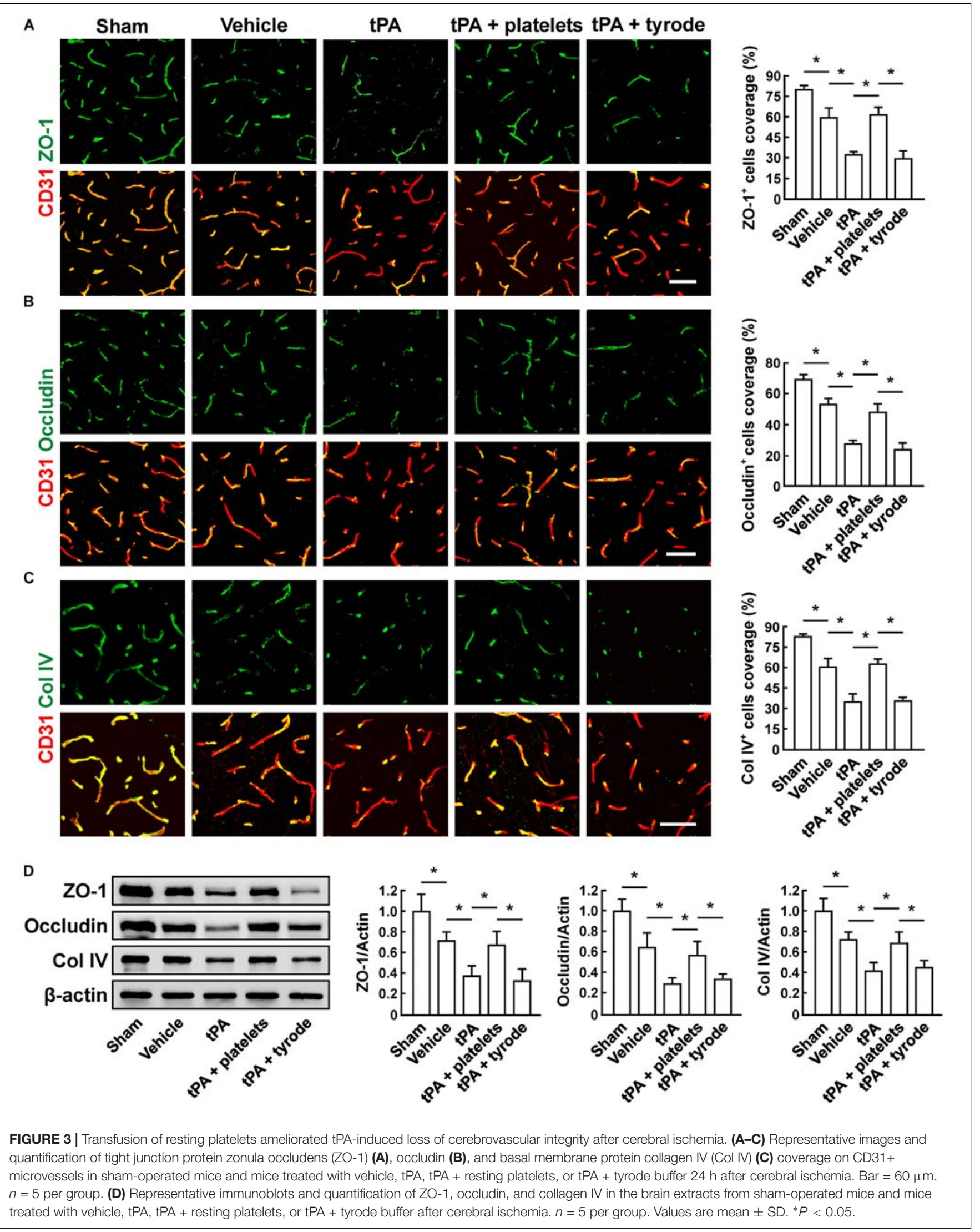



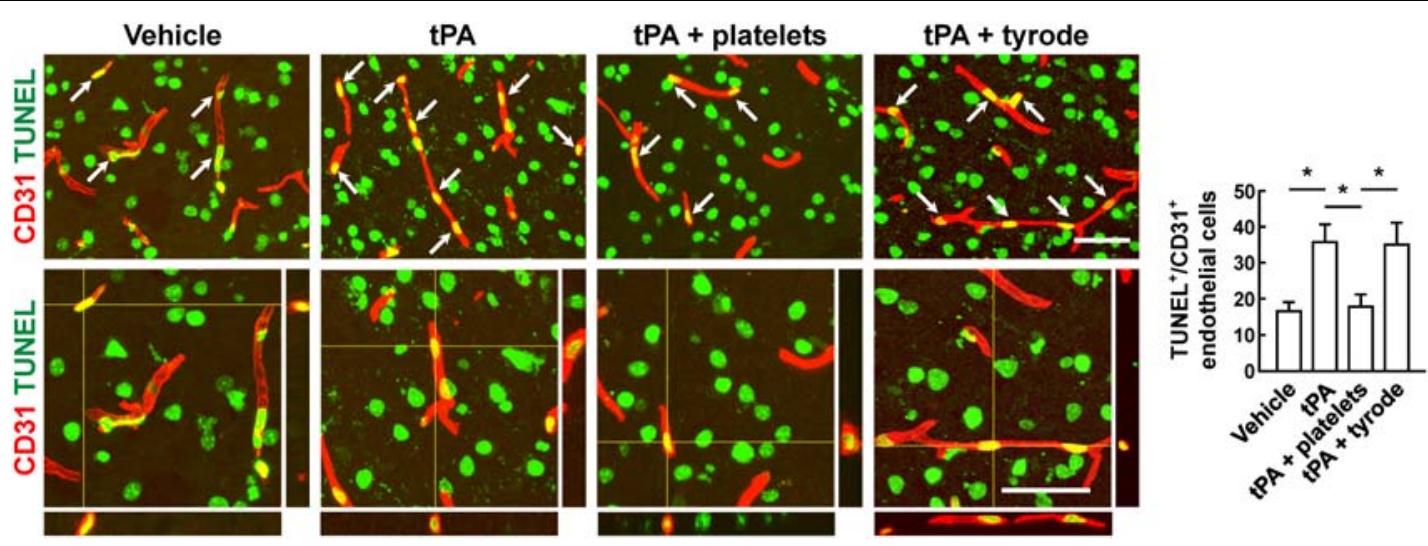

FIGURE 4 | Transfusion of resting platelets reduced tPA-induced loss of vascular endothelial cells after cerebral ischemia. Representative photomicrographs and quantification of TUNEL+/CD31+ endothelial cells in mice treated with vehicle, tPA, tPA + resting platelets, or tPA + tyrode buffer after cerebral ischemia. Bar $=60 \mu \mathrm{m} . n=5$ per group. Values are mean $\pm \mathrm{SD} .{ }^{*} P<0.05$.

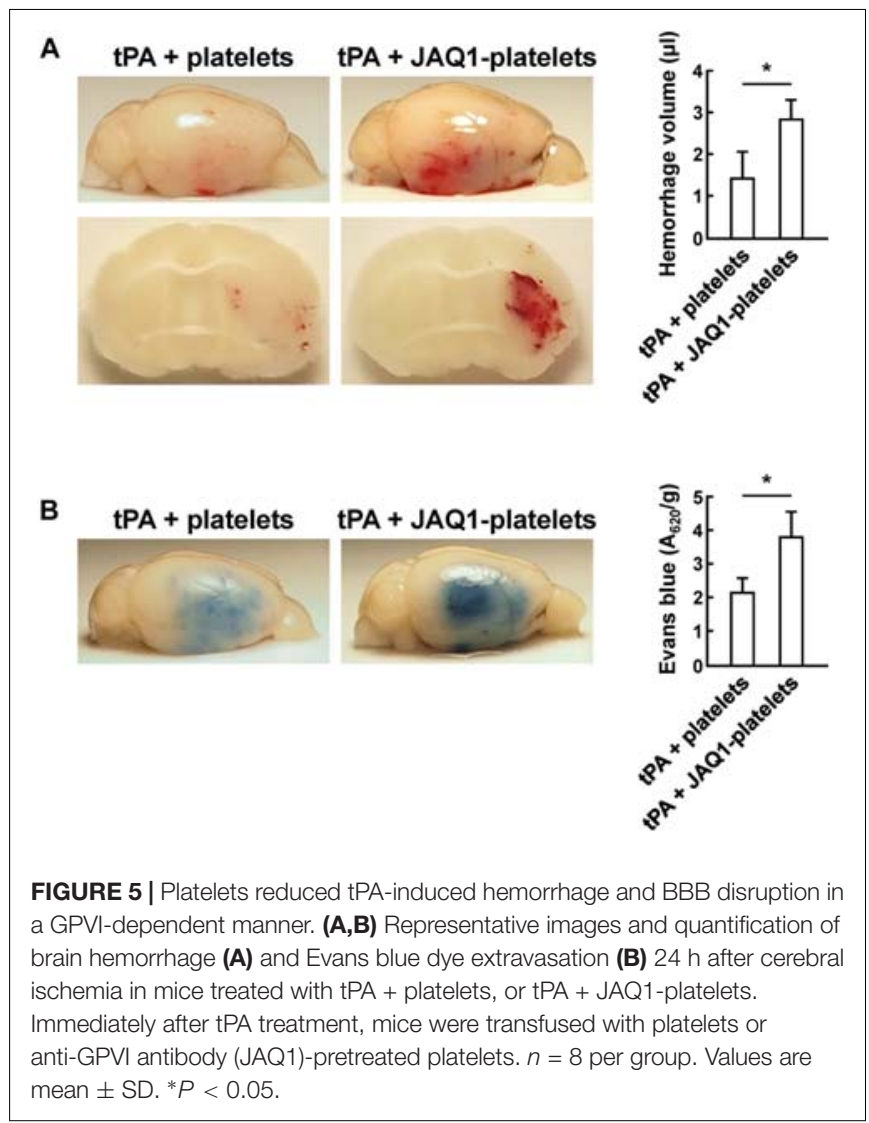

in vitro with a blocking antibody JAQ1 against GPVI before platelet transfusion. Compared to controls, JAQ1-pretreated platelets did not lessen hemorrhage volume (Figure 5A), and the mice with platelets lacking GPVI accumulated significant Evans blue extravasation (Figure 5B). Inhibition of platelet receptor GPVI exhibited no vasculoprotective function, and impaired the ability of resting platelets to reduce tPA-induced hemorrhage and maintain vascular permeability after cerebral ischemia. Our results indicated that the protective effect of platelets in supporting vascular integrity was closely involved in GPVI-dependent signal transduction pathway.

\section{DISCUSSION}

In this study, we showed that resting platelets significantly reduced tPA-mediated loss of cerebrovascular integrity and BBB breakdown, thereby preventing tPA-induced cerebral hemorrhage and improving functional outcomes after cerebral ischemia.

It is well known that platelet adhesion, activation and aggregation are key to thrombosis, which may further lead to thrombotic diseases (Ruggeri, 2002; Mackman, 2008). Historically, antiplatelet therapy for acute stroke was frequently utilized to inhibit platelet activation and aggregation (Bednar and Gross, 1999), which has also been widely combined with thrombolytics in secondary stroke prevention (Luo et al., 2016). However, antiplatelet therapy has been forcefully proven to induce a higher risk of systemic bleeding and intracranial hemorrhage (Serebruany et al., 2004).

In our study, we demonstrated that platelets could effectively prohibit the collagenase-induced $\mathrm{ICH}$ in mice. Consistent with our result above, it has been shown that in some clinical studies, platelet transfusion leads to smaller final hemorrhage size for patients with ICH, which supports a potential treatment of platelet transfusion for ICH (Naidech et al., 2012). Given the evidence that platelets are shown to support vascular development and remodeling, safeguard the lymphatic vessels, secure inflammatory vessels and prevent bleeding at the sites of inflamed organs and tumors (Goerge et al., 2008; Ho-TinNoe et al., 2011). We hypothesize that platelet transfusion could protect brain microvessels integrity, further preventing tPAmediated cerebral hemorrhage. Indeed, our findings provided evidence that platelets played an important role in the maintenance of cerebral vascular integrity, which distinguished from the previous thrombotic propensity of platelets in stroke. 
In our research, we showed that administration with resting platelets decreased the hemorrhage in tPA-treated mice as well as remarkably lowered neurological scores without affecting the infarction volume and intravascular fibrin deposits. However, the life span of transfused platelet in the body is more than 3 days (Odell and McDonald, 1961). To exclude the possibility that transfusion of resting platelets might increase the risk of secondary microvascular thrombosis, further study would be important to examine 3- or 7-day survival rate and neurological scores after platelet transfusion. Notably, after we activated platelets, they lost their ability to prevent bleeding, indicating that prevention of cerebral hemorrhage by platelets might be dependent upon the localized platelet secretions rather than platelet aggregation. Combination treatment with resting platelets and tPA exhibited significant protection of BBB critical components degradation, and inhibition of $\mathrm{BBB}$ disruption as well as reduction of vascular impairment.

The platelet receptor for collagen, GPVI, is believed to be an active participant in preventing excessive blood loss after injury. A previous study found that mice with GPVI deficiency showed defect in hemostasis, as the same results also been confirmed in patients with impaired GPVI expression (Gruner et al., 2004; Hermans et al., 2009). In recent years, GPVI has been found to be pivotal in platelet-endothelium interaction targeting in inflammatory diseases independent of hemostasis and thrombosis, and inhibition of GPVI partially blocks the ability of platelets to prevent inflammatory bleeding (Boulaftali et al., 2013; Gros et al., 2015). Consistently, in acute cerebral ischemia, we demonstrated that inhibition of GPVI impaired the ability of platelets to prevent cerebral hemorrhage. Moreover, platelets deficient in GPVI also showed a defect in blockage of vascular leakage. Together, these data suggest that platelets secure vascular integrity at sites of damaged cerebrovascular maybe mediate via GPVI, and possibly relies on the released platelet granule contents.

\section{CONCLUSION}

In conclusion, we found that resting platelets maintained microvascular integrity, and decreased tPA-associated hemorrhage in acute ischemic stroke. Our data suggest that platelet transfusion would potentially increase the safety of tPA thrombolytic therapy.

\section{REFERENCES}

Arroyo, J. L., Garcia-Marcos, M. A., Lopez, A., Menendez, P., Tabernero, M. D., Sanchez-Abarca, L. I., et al. (2001). Evaluation of a CD61 MoAb method for enumeration of platelets in thrombocytopenic patients and its impact on the transfusion decision-making process. Transfusion 41, 1212-1216. doi: 10.1046/ j.1537-2995.2001.41101212.x

Bednar, M. M., and Gross, C. E. (1999). Antiplatelet therapy in acute cerebral ischemia. Stroke 30, 887-893. doi: 10.1161/01.str.30.4.887

Boulaftali, Y., Hess, P. R., Getz, T. M., Cholka, A., Stolla, M., Mackman, N., et al. (2013). Platelet ITAM signaling is critical for vascular integrity in inflammation. J. Clin. Invest. 123, 908-916. doi: 10.1172/JCI65154

\section{ETHICS STATEMENT}

This study was carried out in accordance with the recommendations of the Animal Care and Use Committee of Institutes of Brain Science, Fudan University. The protocol was approved by the Animal Care and Use Committee of Institutes of Brain Science, Fudan University.

\section{AUTHOR CONTRIBUTIONS}

HL, LW, LL, LK, YC, XY, and XB performed the experiments and analyzed the data. HL, WF, and B-QZ designed the study and wrote the manuscript. All authors read and approved the final manuscript.

\section{FUNDING}

This work was supported by grants from the National Natural Science Foundation of China (General Program 81671156, 31872777, 81471331, and 81873744, Key Program 81530034), the National Key Research and Development Program of China, Ministry of Science and Technology of China (2016YFC1300500-501 and 2016YFC1300500-502), and the Natural Science Foundation of Shanghai (14ZR1401800).

\section{SUPPLEMENTARY MATERIAL}

The Supplementary Material for this article can be found online at: https://www.frontiersin.org/articles/10.3389/fnins. 2019.00338/full\#supplementary-material

FIGURE S1 | (A) Thrombocytopenia was achieved successfully by injection of an anti-mouse GPlb $\alpha$ antibody. Representative density plots of platelets defined by CD61-positivity and sideward light scatter (SSC) characteristics. CD61-FITC was used to stain platelets, expressed as mean fluorescence intensity values (MFI; relative linear units scaled from 0 to $10^{4}$ ), platelet counts expressed as percentage relative to control (100\%) were measured with flow cytometry. $n=8$ per group. Unpaired 2-tailed Student $t$-test. (B) Representative confocal microscopy images and quantitative analysis of fibrin deposits in microvessels from mice treated with vehicle, tPA, tPA + resting platelets, or tPA + tyrode buffer $24 \mathrm{~h}$ after cerebral ischemia. Bar $=60 \mu \mathrm{m} . n=5$ per group. Values are mean $\pm \mathrm{SD} . * P<0.05$.

Bruns, C. J., Solorzano, C. C., Harbison, M. T., Ozawa, S., Tsan, R., Fan, D., et al. (2000). Blockade of the epidermal growth factor receptor signaling by a novel tyrosine kinase inhibitor leads to apoptosis of endothelial cells and therapy of human pancreatic carcinoma. Cancer Res. 60, 2926-2935.

Cai, P., Luo, H., Xu, H., Zhu, X., Xu, W., Dai, Y., et al. (2015). Recombinant ADAMTS 13 attenuates brain injury after intracerebral hemorrhage. Stroke 46, 2647-2653. doi: 10.1161/STROKEAHA.115.00 9526

Cazenave, J.-P., Ohlmann, P., Cassel, D., Eckly, A., Hechler, B., and Gachet, C. (2004). "Preparation of Washed Platelet Suspensions From Human and Rodent Blood," in Platelets and Megakaryocytes Functional Assays, eds J. M. Gibbins and M. P. Mahaut-Smith (Totowa, NJ: Humana Press), 13-28. 
Chen, J. L., Li, Y., Wang, L., Zhang, Z. G., Lu, D. Y., Lu, M., et al. (2001). Therapeutic benefit of intravenous administration of bone marrow stromal cells after cerebral ischemia in rats. Stroke 32, 1005-1011. doi: 10.1161/01.str.32.4. 1005

Deppermann, C., Kraft, P., Volz, J., Schuhmann, M. K., Beck, S., Wolf, K., et al. (2017). Platelet secretion is crucial to prevent bleeding in the ischemic brain but not in the inflamed skin or lung in mice. Blood 129, 1702-1706. doi: 10.1182/blood-2016-12-750711

Fan, M., Xu, H., Wang, L., Luo, H., Zhu, X., Cai, P., et al. (2016). Tissue plasminogen activator neurotoxicity is neutralized by recombinant ADAMTS 13. Sci. Rep. 6:25971. doi: 10.1038/srep25971

Ghoshal, K., and Bhattacharyya, M. (2014). Overview of platelet physiology: its hemostatic and nonhemostatic role in disease pathogenesis. Sci. World J. 2014:781857. doi: 10.1155/2014/781857

Goerge, T., Ho-Tin-Noe, B., Carbo, C., Benarafa, C., Remold-O’Donnell, E., Zhao, B. Q., et al. (2008). Inflammation induces hemorrhage in thrombocytopenia. Blood 111, 4958-4964. doi: 10.1182/blood-2007-11-123620

Goldstein, J. N., Marrero, M., Masrur, S., Pervez, M., Barrocas, A. M., Abdullah, A., et al. (2010). Management of thrombolysis-associated symptomatic intracerebral hemorrhage. Arch. Neurol. 67, 965-969. doi: 10.1001/archneurol. 2010.175

Gros, A., Syvannarath, V., Lamrani, L., Ollivier, V., Loyau, S., Goerge, T., et al. (2015). Single platelets seal neutrophil-induced vascular breaches via GPVI during immune-complex-mediated inflammation in mice. Blood 126, 1017-1026. doi: 10.1182/blood-2014-12-61 7159

Gruner, S., Prostredna, M., Aktas, B., Moers, A., Schulte, V., Krieg, T., et al. (2004). Anti-glycoprotein VI treatment severely compromises hemostasis in mice with reduced alpha2betal levels or concomitant aspirin therapy. Circulation 110, 2946-2951. doi: 10.1161/01.CIR.0000146341.63677.3C

Hacke, W., Kaste, M., Fieschi, C., Toni, D., Lesaffre, E., von Kummer, R., et al. (1995). Intravenous thrombolysis with recombinant tissue plasminogen activator for acute hemispheric stroke. the european cooperative acute stroke study (ECASS). JAMA 274, 1017-1025. doi: 10.1001/jama.274.13.1017

Hermans, C., Wittevrongel, C., Thys, C., Smethurst, P. A., Van Geet, C., and Freson, K. (2009). A compound heterozygous mutation in glycoprotein VI in a patient with a bleeding disorder. J. Thromb. Haemost. 7, 1356-1363. doi: 10.1111/j.1538-7836.2009.03520.x

Ho-Tin-Noe, B., Demers, M., and Wagner, D. D. (2011). How platelets safeguard vascular integrity. J. Thromb. Haemost. 9, 56-65. doi: 10.1111/j.1538-7836.2011. 04317.x

Ho-Tin-Noe, B., Goerge, T., Cifuni, S. M., Duerschmied, D., and Wagner, D. D. (2008). Platelet granule secretion continuously prevents intratumor hemorrhage. Cancer Res. 68, 6851-6858. doi: 10.1158/0008-5472.CAN-080718

Jauch, E. C., Saver, J. L., Adams, H. P. Jr., Bruno, A., Connors, J. J., Demaerschalk, B. M., et al. (2013). Guidelines for the early management of patients with acute ischemic stroke: a guideline for healthcare professionals from the american heart association/american stroke association. Stroke 44, 870-947. doi: 10.1161/ STR.0b013e318284056a

Lee, R. H., and Bergmeier, W. (2016). Platelet immunoreceptor tyrosine-based activation motif (ITAM) and hemITAM signaling and vascular integrity in inflammation and development. J. Thromb. Haemost. 14, 645-654. doi: 10.1111/ jth. 13250

Luo, S., Zhuang, M., Zeng, W., and Tao, J. (2016). intravenous thrombolysis for acute ischemic stroke in patients receiving antiplatelet therapy: a systematic review and meta-analysis of 19 studies. J. Am. Heart Assoc. 5:e03242. doi: 10.1161/JAHA.116.003242

Mackman, N. (2008). Triggers, targets and treatments for thrombosis. Nature 451, 914-918. doi: 10.1038/nature06797

Mancuso, M. E., and Santagostino, E. (2017). Platelets: much more than bricks in a breached wall. Br. J. Haematol. 178, 209-219. doi: 10.1111/bjh.14653
Matsushita, H., Hijioka, M., Hisatsune, A., Isohama, Y., Shudo, K., and Katsuki, H. (2011). A retinoic acid receptor agonist Am80 rescues neurons, attenuates inflammatory reactions, and improves behavioral recovery after intracerebral hemorrhage in mice. J. Cereb. Blood Flow Metab. 31, 222-234. doi: 10.1038/ jcbfm. 2010.80

Naidech, A. M., Liebling, S. M., Rosenberg, N. F., Lindholm, P. F., Batjer, H. H., and Kwaan, H. C. (2012). Early platelet transfusion improves platelet activity and may improve outcomes after intracerebral hemorrhage response. Neurocrit. Care 17, 156-157. doi: 10.1007/s12028-012-9698-9699

Nieswandt, B., and Watson, S. P. (2003). Platelet-collagen interaction: is GPVI the central receptor? Blood 102, 449-461. doi: 10.1182/blood-2002-12-3882

Odell, T. T., and McDonald, T. P. (1961). Life span of mouse blood platelets. Proc. Soc. Exp. Biol. Med. 106, 107-108. doi: 10.3181/00379727-106-26252

Rasche, H. (2001). Haemostasis and thrombosis: an overview. Eur. Heart J. Suppl. 3(Suppl. Q), Q3-Q7. doi: 10.1016/S1520-765X(01)90034-90033

Ribatti, D., and Crivellato, E. (2007). Giulio bizzozero and the discovery of platelets. Leuk. Res. 31, 1339-1341. doi: 10.1016/j.leukres.2007.02.008

Roth, G. A., Huffman, M. D., Moran, A. E., Feigin, V., Mensah, G. A., Naghavi, M., et al. (2015). Global and regional patterns in cardiovascular mortality from 1990 to 2013. Circulation 132, 1667-1678. doi: 10.1161/CIRCULATIONAHA. 114.008720

Ruggeri, Z. M. (2002). Platelets in atherothrombosis. Nat. Med. 8, 1227-1234. doi: $10.1038 / \mathrm{nm} 1102-1227$

Serebruany, V. L., Malinin, A. I., Eisert, R. M., and Sane, D. C. (2004). Risk of bleeding complications with antiplatelet agents: meta-analysis of 338,191 patients enrolled in 50 randomized controlled trials. Am. J. Hematol. 75, 40-47. doi: 10.1002/ajh.10451

Suzuki, Y., Nagai, N., and Umemura, K. (2016). A Review of the mechanisms of blood-brain barrier permeability by tissue-type plasminogen activator treatment for cerebral ischemia. Front. Cell Neurosci. 10:2. doi: 10.3389/fncel. 2016.00002

Vaiyapuri, S., Jones, C. I., Sasikumar, P., Moraes, L. A., Munger, S. J., Wright, J. R., et al. (2012). Gap junctions and connexin hemichannels underpin hemostasis and thrombosis. Circulation 125, 2479-2491. doi: 10.1161/ CIRCULATIONAHA.112.101246

Wang, L., Fan, W., Cai, P., Fan, M., Zhu, X., Dai, Y., et al. (2013). Recombinant ADAMTS13 reduces tissue plasminogen activator-induced hemorrhage after stroke in mice. Ann. Neurol. 73, 189-198. doi: 10.1002/ana.23762

Whiteley, W. N., Slot, K. B., Fernandes, P., Sandercock, P., and Wardlaw, J. (2012). Risk factors for intracranial hemorrhage in acute ischemic stroke patients treated with recombinant tissue plasminogen activator: a systematic review and meta-analysis of 55 studies. Stroke 43, 2904-2909. doi: 10.1161/STROKEAHA. 112.665331

Xu, H. C., Cao, Y. L., Yang, X., Cai, P., Kang, L. J., Zhu, X. M., et al. (2017). ADAMTS13 controls vascular remodeling by modifying VWF reactivity during stroke recovery. Blood 130, 11-22. doi: 10.1182/blood-2016-10-747089

Zhao, B.-Q., Chauhan, A. K., Canault, M., Patten, I. S., Yang, J. J., Dockal, M., et al. (2009). von Willebrand factor-cleaving protease ADAMTS13 reduces ischemic brain injury in experimental stroke. Blood 114, 3329-3334. doi: 10.1182/blood2009-03-213264

Conflict of Interest Statement: The authors declare that the research was conducted in the absence of any commercial or financial relationships that could be construed as a potential conflict of interest.

Copyright (C) 2019 Luo, Wei, Lu, Kang, Cao, Yang, Bai, Fan and Zhao. This is an open-access article distributed under the terms of the Creative Commons Attribution License (CC BY). The use, distribution or reproduction in other forums is permitted, provided the original author(s) and the copyright owner(s) are credited and that the original publication in this journal is cited, in accordance with accepted academic practice. No use, distribution or reproduction is permitted which does not comply with these terms. 